\title{
Dipole relaxation losses in DNA
}

\author{
M. Briman, N.P. Armitage, E. Helgren, and G. Grüner \\ Department of Physics and Astronomy, University of California, Los Angeles, CA 90095
}

(August 22, 2018)

\begin{abstract}
The electrodynamic response of DNA in the millimeter wave range is investigated. By performing measurements under a wide range of humidity conditions and comparing the response of single strand DNA and double strand DNA, we show that the appreciable AC conductivity of DNA is not due to photon activated hopping between localized states, but instead due to dissipation from dipole motion in the surrounding water helix. Such a result, where the conductivity is due to the constrained motion of overdamped dipoles, reconciles the vanishing DC conductivity of DNA with the considerable $\mathrm{AC}$ response.
\end{abstract}

PACS numbers: 87.14.Gg, 72.80.Le

The electrical conductivity of DNA has been a topic of much recent interest and controversy [1]. Measurements from different groups have reached a variety of conclusions about the nature of charge transport along the double helix. DNA has been reported to be metallic [2], semiconducting [3], insulating [4,5], and even a proximity effect induced superconductor [6]. However, questions have been raised with regards to the role played by electrical contacts, length effects, and the manner in which electrostatic damage, residual salt concentrations, and other contaminations may have affected these results [1]. More recent measurements, where care was taken to both establish a direct chemical bond between $\lambda$-DNA and $\mathrm{Au}$ electrodes and also control the excess ion concentration, have given compelling evidence that the DC resistivity of the DNA double helix over long length scales $(<10 \mu \mathrm{m})$ is very high indeed $\left(\rho>10^{6} \Omega-\mathrm{cm}\right)$ [7]. Such DC measurements contrast with recent contactless AC measurements that have shown that there is appreciable conductivity at microwave and far-infrared frequencies $[8,9]$ the magnitude of which approaches that of a welldoped semiconductor [10].

Previously, the AC conductivity in DNA was found to be well parameterized as a power-law in $\omega[8,9]$. Such a dependence can be a general hallmark of AC conductivity in disordered systems with photon assisted hopping between random localized states [11] and led to the reasonable interpretation that intrinsic disorder, counterion fluctuations, and possibly other sources created a small number of electronic states on the base pair sequences in which charge conduction could occur. However, such a scenario would lead to thermally activated hopping conduction between localized states and is thus inconsistent with the very low DC conductivity [7]. A number of outstanding issues arise: Are there localized regions along the helix where a continuous conducting path is not present, but still AC hopping between localized states over distances of a few base pairs can occur? Are there sensitive length dependencies in the DNA strands? Is there a difference between between the samples of various groups? Perhaps different charge conduction mechanisms play a role at finite frequency.

To the end of resolving some of these matters, we have performed $\mathrm{AC}$ conductivity experiments in the millimeter wave range under a wide range of humidity conditions. We show that the appreciable AC conductivity of DNA in the microwave and far infrared regime should not be viewed as some sort of hopping between localized states and is instead likely due to dissipation in the dipole response of the water molecules in the surrounding hydration layer. It can be well described by a Debye-like relaxation of water molecules in the surrounding water helix. At low humidities the response is well modelled by considering the rotation of single water molecules in the structural water layer. As the number of water molecules per base pair increases, dissipation due to the collective motion of water dipoles increases, until eventually the conductivity resembles that of bulk water. By measuring both single strand (ssDNA) and double strand DNA (dsDNA) over a wide range of humidities we are able to show that, at least in principle, all the $\mathrm{AC}$ conductivity of DNA can be assigned to relaxation losses of water dipoles. This result reconciles the apparent complete lack of DC conductivity with the appreciable AC response.

Double stranded DNA films were obtained by vacuum drying of $7 \mathrm{mM}$ PBS solution containing $20 \mathrm{mg} / \mathrm{ml}$ sodium salt DNA extracted from calf thymus and salmon testes (Sigma D1501 and D1626). The results were found to be independent of the use of calf or salmon DNA. Our choice for these concentrations deserves further explanation. It is well known that at a given temperature double helical conformation of DNA can exist in solution only with a certain concentration of positive ions. Excess salt cannot be removed by vacuum drying, so large amounts of residual salt in films could introduce significant errors in conductivity, due to both the ionic conduction of the salt itself and its additional hydration during humidity changes. Melting temperature calculations $[12,13]$ for 
long native pieces of DNA with C-G content around $40 \%$ show that $2-10 \mathrm{mM}$ concentration of sodium cations is enough to stabilize the double helix at room temperature. Films were prepared with differing salt amounts and it was found that as long as the excess salt mass fraction is kept between $2-5 \%$ the final results were not significantly affected. In order to improve the DNA/salt mass ratio we used a high concentration of DNA, but $20 \mathrm{mg} / \mathrm{ml}$ appears to be the limit. Higher concentrations makes it difficult for DNA fibers to dissolve and the solution becomes too viscous, which prevents producing the flat uniform films which are of paramount importance for the quasi-optical resonant technique. Single stranded DNA films were prepared from the same original solution as the double stranded ones, with preliminary heating up to 95 $\mathrm{C}$ for 30 minutes and fast cooling down to $4 \mathrm{C}$. In both dsDNA and ssDNA cases the conformational state was checked by fluorescent microscope measurements. The dry films were 20 to 30 microns thick and were made on top of $1 \mathrm{~mm}$ thick sapphire windows. Immediately after solution deposition onto the sapphire substrates the air inside the viscous solution was expelled by vacuum centrifuging at $500 \mathrm{~g}$, otherwise the evaporation process causes the formation of air bubbles that destroy the film uniformity.

The AC conductivity was measured in the millimeter spectral range. Backward wave oscillators (BWO) in a quasi-optical setup (100 Ghz - $1 \mathrm{THz}$ ) were employed as coherent sources in a transmission configuration. This difficult to access frequency range is particularly relevant as it corresponds to the approximate expected time frame for relaxation processes in room temperature liquids (1$10 \mathrm{ps}$ ). Importantly, it is also below the energy range where one expects to have appreciable structural excitations. The technique and analysis are well established [14]. We utilize the fact that for plane waves incident normally on a slab of material, transmission resonances occur when the slab is an integer number of half wavelengths. Thus, using $\mathrm{a} \approx 1 \mathrm{~mm}$ sapphire disc as a substrate, resonances occurred approximately every $50 \mathrm{GHz}$. Having analyzed the transmission through the sapphire alone prior to mounting the sample, the optical properties of the substrate were well characterized. Thus using a two-layer transmission model, each resonance can be analyzed to extract the optical properties of the DNA film, allowing for a $1.5 \mathrm{~cm}^{-1}$ resolution of the spectra.

Samples were measured at room temperature at several fixed humidity levels which were maintained by putting them in a hermetically sealed environment with a saturated salt solution [15]. The change in thickness and mass of the DNA films at different humidities were tracked by separate measurements within a controlled environment for each sample in a glove box. The total number of water molecules per nucleotide $A$ can be correlated to the relative humidity $\mathrm{x}(\mathrm{x}=0-1)$ through the so-called Branauer-Emmett-Teller (BET) equation [16]

$$
A=\frac{B C x}{(1-x)(1-x+C x)} .
$$

The constant $B$ is the maximum number of water molecules in the first layer sites. According to the statistical formulation of the BET equation by Hill [17], mobile water molecules within the double helix can be characterized as 2 types. The first are ones within the initial hydration layer, which are directly attached to DNA and have a characteristic binding energy $\epsilon_{1}$. Water molecules of the second and all other layers can be approximated as having a binding energy $\epsilon_{L}$. To a good approximation this $\epsilon_{L}$ can be taken to be that of bulk water. These parameters enter into the BET equation through the expression for $C$ which equals $D e^{\left(\frac{\epsilon_{1}-\epsilon_{L}}{k T}\right)}$ where $D$ is related to the partition function of water. Also we should note that there is, in actuality, a structural 0-th layer of water molecules, containing 2.5-3 water molecules per nucleotide that cannot be removed from the helix under typical conditions [18].

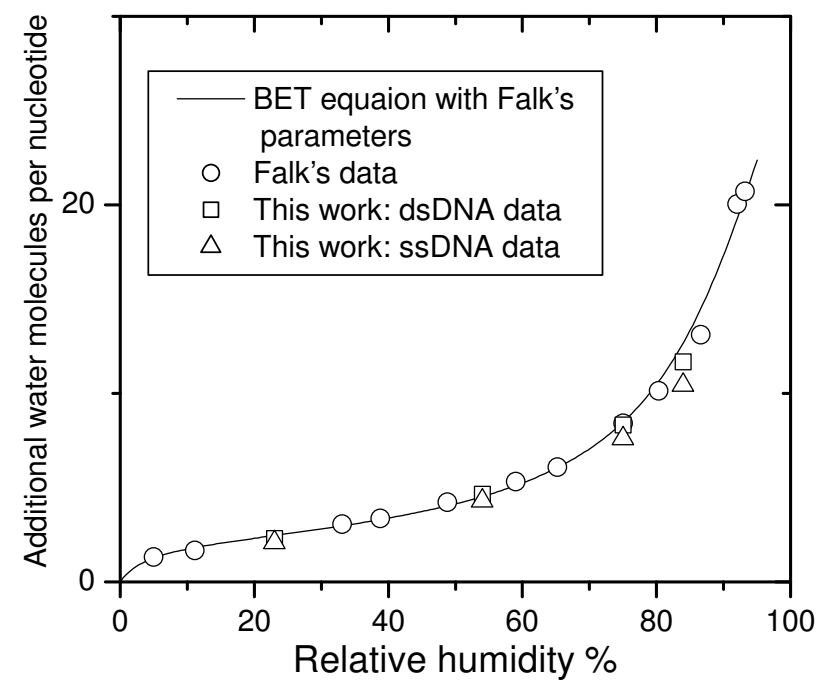

FIG. 1. Absorbtion of water molecules per nucleotide as a function of humidity. The data represented by the open circles is taken from Falk et. al.

That it is reasonable that the mobile water layers of DNA can be modelled by distinguishing 2 different sets of water parameters was first established by Falk et al.'s [15] use of the BET equation to describe the hydration of sodium and lithium DNA salts from calf thymus and salmon testes. They found good agreement between experimental data and theory with constants $B=2.2$ and $C=20$. We performed a similar hydration study of our dsDNA and ssDNA films; as shown in Fig. 1 the hydration of our films are perfectly consistent with Falk's result. Note that there is no appreciable difference in the hydration between dsDNA and ssDNA.

In Fig. 2 we present data for the extracted $\sigma_{1}(\omega)$ of 
both dsDNA and ssDNA thin films. One can see that in both cases, the conductivity is an increasing function of frequency. Since the conductivity is also an increasing function of humidity, one may wish to try to seperate the relative contributions of charge motion along the DNA backbone from that of the surrounding water molecules.

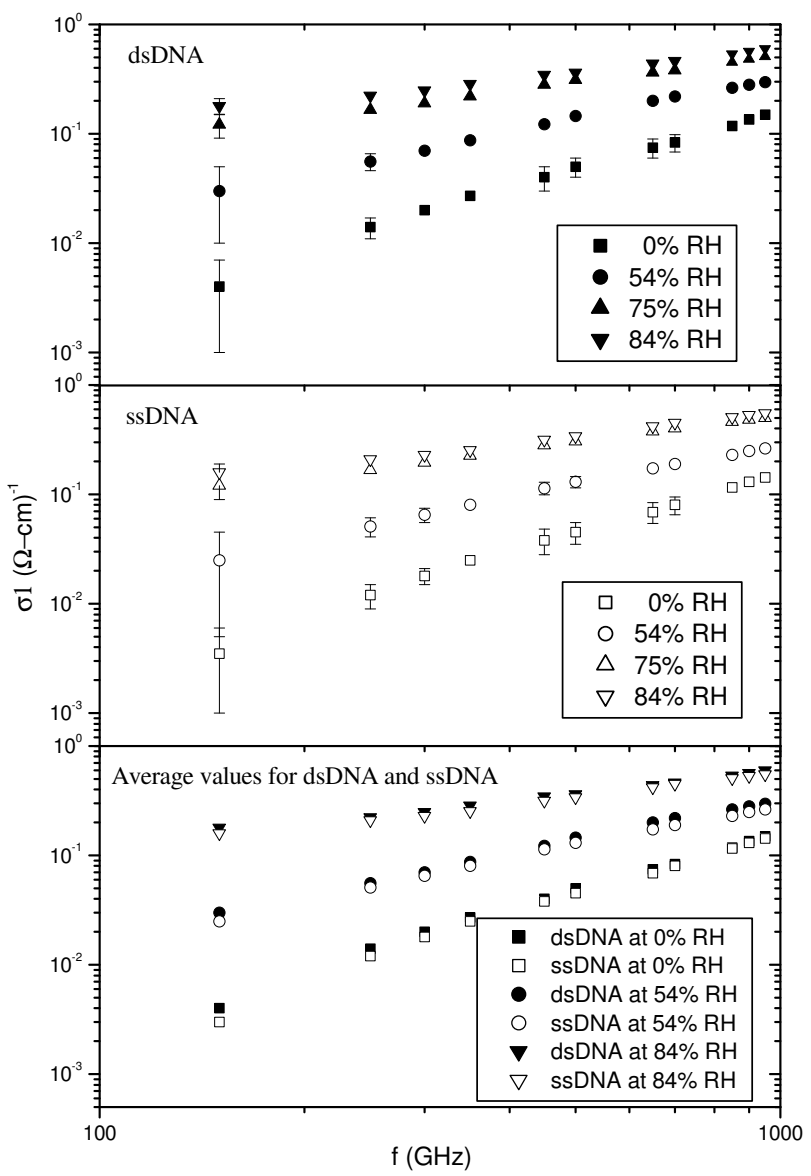

FIG. 2. Frequency dependence of the conductivity of calf thymus DNA at different relative humidity levels. (a) Double stranded DNA (b) Single stranded DNA (c) A comparison of conductivity between single and double stranded DNA.

First, one can consider that there should be two main effects of hydration in our dsDNA films. There is the hydration itself, where water molecules are added in layers to the double helix; this is well described by BET equation [16]. Additionally, the conformational state of dsDNA also changes as a function of humidity. For example, sodium salt calf thymus DNA is in a B-like disordered form at humidities from $0-40 \%$, above which it transfers to the A form, and finally to a well ordered B-form at humidities higher than $80 \%[19,20]$. Additional water molecules certainly contribute to the increase in conductivity, but at high humidities there is the possibility that some of the conduction might be due to an increase in electron transfer along the dsDNA helix in the ordered
$B$ form. However since such an effect would be much reduced in disordered and denaturalized ssDNA films and since Fig. 2 shows that to within the experimental uncertainty the conductivity of dsDNA and ssDNA in the millimeter wave range is identical, it is most natural to suggest that water is the major contribution to the $\mathrm{AC}$ conductivity. From this comparison of dsDNA and ssDNA, we find no evidence for charge conduction along the DNA backbone.

In Fig. 3 we plot the the conductivity $\sigma_{1}$ of the DNA films normalized by the expected volume fraction of water molecules including both the hydration layers plus the structural water. Although this normalization reduces the spread in the thin film conductivity at the lowest frequencies it does not reduce it to zero, showing that if the largest contribution to the conductivity comes from water, the character of its contribution changes as a function of humidity.

The complex dielectric constant of bulk water has been shown to be well described by a biexponential Debye relaxation model [21-23], where the first relaxation process [21], characterized by a time scale $\tau_{D}=8.5 \mathrm{ps}$, corresponds to the collective motion of tetrahedral water clusters, and the second from faster single molecular rotations [24] with a time scale $\tau_{F}=170$ fs. For bulk water, the contribution of each relaxation process is determined by the static dielectric constant $\epsilon_{S}(T)=$ $87.91 e^{-0.0046 T\left[{ }^{\circ} C\right]}, \epsilon_{1}=5.2$, and the dielectric constant at high frequencies $\epsilon_{\infty}=3.3$.

$$
\widehat{\epsilon}(w)=\epsilon_{\infty}+\frac{\epsilon_{S}-\epsilon_{1}}{1+i \omega \tau_{D}}+\frac{\epsilon_{1}-\epsilon_{\infty}}{1+i \omega \tau_{F}}
$$

When applying Eq. 2 to the dipole relaxation losses of DNA, one expects that the relative contributions of the two frequency dependent terms will change as increasing humidity increases the average effective coordinate number. For instance, at $0 \%$ humidity it is reasonable to assume that the first term which is due to the collective motion of water clusters, cannot play a role as the structural water is not tetrahedrally coordinated. For high hydration levels, where multiple water layers exist around the dipole helix, the relaxation losses of the water layer may approach those of bulk water. We can compare the above equation using the independently known values [21] for $\tau_{D}, \epsilon_{S}, \tau_{F}$ and $\epsilon_{1}$ to the experimental data normalized to the expected volume fraction of the water from the independently determined water uptake curves shown in Fig. 1. In Fig. 3, along with the experimental data at two representative humidity levels, two theoretical curves for $0 \%$ and $100 \%$ humidity are plotted. With the only two assumptions being that at $0 \%$ humidity, the sole relaxational losses come from singly coordinated water molecules in the structural water layer and that it is only at higher humidity levels where the collective losses can gradually play a greater role, the theoretical curves provide a very good fit to the data over almost all of 
the measured frequency range. At low humidity the data is well matched by the theory incorporating only single molecule rotations. At high humidity, the data begins to approach the behavior of 'free' water. For these two limits the theoretical curves have no free parameters.

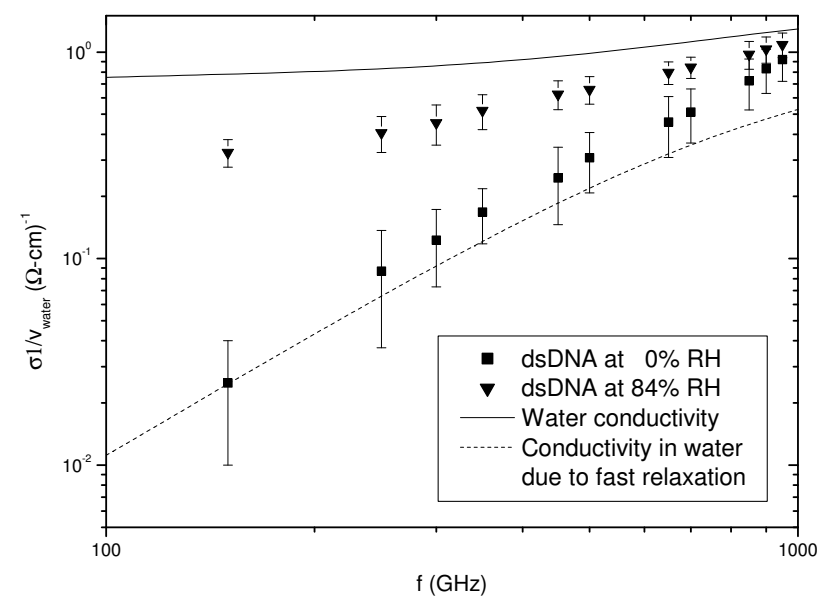

FIG. 3. Conductivity of dsDNA and ssDNA films normalized by the volume fraction of all water molecules (structural plus hydration layer). For clarity, only $0 \%$ and $84 \%$ humidities are shown. The solid line represents the conductivity of pure water as modelled by the biexponential Debye model using the parameters of Ronne et al. The dashed line shows just the contribution from single water molecule relaxation.

The only appreciable discrepancy between theory and experiment is the high frequency data at low humidity, where the biexponential Debye model underestimates the conductivity. This may be due to a number of reasons. At very low relative humidities it is possible for the ionic phosphate groups on the DNA backbone to form stable dihydrates which may give their own contribution to relaxation losses through their additional degree of freedom [15]. Alternatively, it may also be that at higher frequencies for low hydration samples, the weak restoring force from charge-dipole interaction in the structural water layer begins to become more significant and our biexponential Debye model becomes less applicable.

In conclusion, we have found that the considerable AC conductivity of DNA can be largely ascribed to relaxational losses of the surrounding water dipoles. The conductivity of ssDNA and dsDNA was found to be identical to within the experimental error, indicating that there is essentially no charge conduction along the DNA backbone itself. The conclusion that the observed conductivity derives from the water layer is supported by the fact that, over much of the range, it can be well described by a biexponential Debye model, where the only free parameter is the relative contributions of single water molecule and tetrahedral water cluster relaxation modes. Generally speaking, because many large biomolecules have sur- rounding water layers, a result such as ours shows that one must be aware of the possibility of such relaxation losses when investigating the electrodynamic response of such systems.

We would like to thank K. Greskoviak for help with sample preparation. The research at UCLA was supported by the National Science Foundation grant DMR0077251 .

[1] C. Dekker and M. Ratner, Physics World 14, 29 (2001).

[2] H. W. Fink and C. Schonenberger, Nature 398, 407 (1999).

[3] D. Porath et al., Nature 403, 635 (2000).

[4] E. Braun et al., Nature 391, 775 (1998).

[5] P. J. de Pablo et al., Phys. Rev. Lett. 85, 4992 (2000).

[6] A. Y. Kasumov et al., Science 291, 280 (2001).

[7] Zhang et al. Phys. Rev. Lett. 89, 198102 (2002).

[8] P. Tran, B. Alavi and G. Grüner, Phys. Rev. Lett. 85, 1564 (2000).

[9] E. Helgren et al. cond-mat/0111299.

[10] E. Helgren, N.P. Armitage, and G. Grüner, Phys. Rev. Lett. 89, 246601 (2002).

[11] A. L. Efros and B. I. Shklovskii, J. Physics C 8, L49 (1975).

[12] E. Breslauer et al., Proc. Nat. Acad. Sci. 83, 3746 (1986).

[13] N. Sugimoto et al., Nucl. Acids Res. 24, 4501 (1996).

[14] A. Schwartz et al., Rev. Sci. Instrum. 66, 2943 (1995).

[15] M. Falk, K. Hartman, R. Lord, J. Am. Chem. Soc. 84, 3843 (1962).

[16] S. Braunauer, P. Emmett, E. Teller, J. Am. Chem. Soc. 80, 309 (1938).

[17] T. L. Hill, J. Chem. Phys. 14, 263 (1946).

[18] N. Tao and S. Lindsay, Biopolymers 28, 1019 (1989).

[19] V.Y. Maleev et al., Biofizika 38, 768, (1993).

[20] S. M. Lindsay et al. Biopolymers, 27, 1015, (1988).

[21] C. Ronne et al., J. Chem. Phys. 107, 5319 (1997)

[22] J. Kindt, C. Schmuttenmaer, J. Phys. Chem. 100, 10373 (1996).

[23] J. Barthel, R. Buchner, Pure and Appl. Chem. 63, 1473 (1991).

[24] N. Agmon, J. Phys. Chem. 100, 1072 (1996). 